than 3000 feet. But our knowledge is still far from complete, and all who may obtain observations on this subject should follow Mr. Blathwayt's example by putting the occurrence on record (even if the species is not identified).

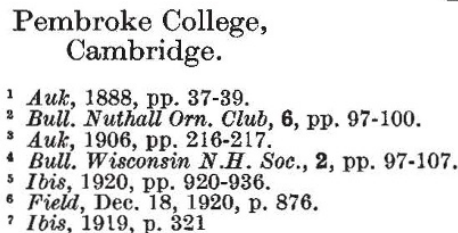

\section{Ultra-Violet Absorption and Raman Effect for Hydrazine.}

THE ultra-violet absorption in hydrazine vapour, $\mathrm{N}_{2} \mathrm{H}_{4}$ (saturation pressure over the liquid at room temperature), consists of five or six nearly equidistant intensity maxima at about 2326, 2320, 2276, 2250, 2225 A., followed by continuous absorption, beginning at about $2200 \mathrm{~A}$. and extending into the whole quartz region. ${ }^{1}$ The band absorptions are entirely devoid of rotation structures at $5.4 \mathrm{~A}$. per $\mathrm{mm}$. dispersion.

To obtain some knowledge about the mechanism of dissociation shown at $2200 \mathrm{~A}$., I have also photographed the Raman spectrum of the same substance using a column of liquid $1 \mathrm{~cm}$. in diameter and $10 \mathrm{~cm}$. in length, irradiated by a suitably filtered mercury are so that only the radiations at $4047 \mathrm{~A}$. and $4359 \mathrm{~A}$. are effective (details of the experiment to be published in the Scientific Papers of this Institute).

Two sets of five sharp modified lines are observed under low dispersion, simply displaced by the separation of the two exciting lines, with the following frequency shifts from their respective lines of origin :

$$
\begin{array}{r}
\Delta \nu\left(\mathrm{cm} .^{-1}\right) a, 900 \text { (weak), } 1120, \\
b, 3212,3289,3339 .
\end{array}
$$

The $b$ lines are nearly of the same intensity and agree very well with the triplet observed in liquid ammonia $\left(\mathrm{NH}_{3}\right)$ by Daure ${ }^{2}$ and others. They represent undoubtedly the internal vibration frequencies of the N-H or $\mathrm{NH}_{2}$ group, the last one corresponding to the absorption centre of the $3 \mu$ band of ammonia $\left(3337 \mathrm{~cm}^{-1}\right)$.

Now, Dadieu and Kohlrausch ${ }^{3}$ interpret Daure's line of lower frequency, $1070 \mathrm{~cm}^{-1}$ of ammonia, as due to the single bond vibration in the polymerised molecule $\mathrm{H}_{3} \mathrm{~N}-\mathrm{NH}_{3}$ with quadrivalent nitrogen supposed to exist in liquid ammonia. If this is true, my 1120 line is safely attributed to the N-H (single bond) vibration in the hydrazine molecule.

The hydrazine used was prepared from the hydrate $\mathrm{N}_{2} \mathrm{H}_{4} \cdot \mathrm{H}_{2} \mathrm{O}$, and the weak line at $900 \mathrm{~cm}^{-1}$ is probably due to hydrazine hydrate molecules still remaining. This point I hope to verify with pure hydrate, but at the moment the substance is rather difficult to obtain.

It is not difficult to imagine that the N-N binding is very much loosened in an excited gaseous state, and the $500 \mathrm{~cm}^{-1}$ vibration frequency observed in ultra-violet absorption may arise from this. The dissociation of hydrazine vapour by 5.5 volt light absorption therefore seems to be effected by the breaking of the excited molecule in the middle. Considering the sharpness of the spectrum, the bands preceding may be regarded as a structureless predissociation spectrum.

Institute of Physical and

Chemical Research, Tokyo, April 17.

1 Sci. Pap. Inst. Phys. Chem. Res., 15, 166 ; 1931

1 Sci. Pap. Anst. Phys. Chem. Res., 188,$61 ; 1929$.

2.R. Acad. Sci., 188, 61; 192

No. 3212, VoL. 127]
Crystal Structure of Chromium Trioxide.

SMaLl thin red needles, heavily striated down the prism, were prepared by adding concentrated sulphuric acid to a cold concentrated solution of potassium dichromate. The crystals were sealed up separately in thin glass tubes to prevent deliquescence. Measurements on an ionisation spectrometer and from oscillation photographs gave the value of the cell size as $a=8.50 \mathrm{~A} ., b=4.73 \mathrm{~A} ., c=5.72 \mathrm{~A}$., the probable error being not greater than \pm 1 per cent. The density given by Groth is $2 \cdot 73-2 \cdot 82$ and there are therefore 4 molecules per cell. The axial ratios given by Nordenskjöld ${ }^{1}$ are $a: b: c=1: 0.69: 0.63$. The relation between his ratios and ours is $a: b: c$ (Wooster) $=$ $a: \frac{3}{4} b: c$ (Nordenskjöld). The only face from which he could obtain the ratio $a: b$ should have been indexed (230) instead of (120). All the specimens which we have examined up to the present are mimetic twins on (110), closely simulating hexagonal symmetry.

Ionisation and photographic investigations show. the following halvings :

$$
(h k l) \text { when }(h+k) \text { is odd. }
$$$$
(0 k l) \text { when } l \text { is odd. }
$$

These suggest the space-group $Q_{h}^{17}-C \mathrm{cmm}$ and the co-ordinates of the chromiums are as follows, the centre of symmetry being taken as origin: $u, 0, \frac{1}{4}$; $\bar{u}, 0, \overline{\frac{1}{4}} ; \frac{1}{2}+u, \frac{1}{2}, \frac{1}{4} ; \frac{1}{2}-u, \frac{1}{2}, \overline{\frac{1}{4}}$. The value of $u$ is doubtful, because the intensities are disturbed by the twinning, and fall off very rapidly, but $u$ is probably about $\frac{1}{9}$. The observed halvings appear to require a structure consisting of tetrahedra of $\mathrm{CrO}_{4}$ linked together in chains parallel to the $c$ axis. This would explain the cleavages, which are very well marked parallel to this direction and tend to cause the crystal to break up into fine needles.

The work will shortly be continued at low temperatures and published in the Zeitschrift für Kristallographie.

W. A. Wooster.

$$
\begin{aligned}
& \text { Mineralogical Laboratory, } \\
& \text { Cambridge, } \\
& \text { May 6. } \\
& \text { ' A. E. Nordenskjöld, Pogg. Ann. Phy. u. Chem., 114, } 622 \text {; } 1861 .
\end{aligned}
$$

\section{Deep Focus Earthquakes.}

IN a letter published in NaTURE of Mar. 28 (p. 486), Mr. F. J. Scrase, referring to an earthquake which was registered at Kew Observatory on $\mathrm{Feb}$. 20, explained briefly how it was possible to recognise the abnormal depth of focus and to make an estimate of this depth. Confirmation of the deep focus of this earthquake has now appeared in a Seismological Bulletin issued by the Jesuit Seismological Association of America.

From the fact that the primary pulse (or $P$ wave) reached Kew, Pasadena, and Riverview almost simultaneously, it is deduced that the epicentre of the earthquake was in latitude $44^{\circ} \mathrm{N}$. and longitude $135^{\circ}$ E., about 200 miles from Vladivostok. At every station for which the records have been examined, the interval between the times of arrival of the $P$ and $S$ waves was abnormally short for the distance from the epicentre. Moreover, the arrival time at La Paz of $P^{\prime}$ (the horizontal wave which passes through the earth's central core) is about one minute early, and, as $\mathrm{La} \mathrm{Paz}$ is at an epicentral distance of about $150^{\circ}$, this early arrival strongly supports the other evidence for deep focus.

Kew Observatory, F. J. W. WhIPPLE. April 30. 